while $73 \%$ of low risk pregnancies developed intrapartum risk factors such as maternal pyrexia, tachycardia and meconium. Only $7.3 \%$ experienced significant intrapartum events (abruption, shoulder dystocia). $37 \%$ of all neonates were admitted to the neonatal unit with $62 \%$ of them needing intubation. $92 \%$ of babies needing intubation were from low risk pregnancies who developed intrapartum risk factors, most of which were not recognised and acted upon appropriately.

Conclusion Low AS in term neonates seems to be associated with new onset of intrapartum risk factors rather than pre-exiting risk factors. Birth attendants should pay more attention to early recognition of risk factors through continuous risk assessment, and intervene appropriately, in order to prevent unexpected poor neonatal outcome.

\section{PL.77 COMPLIANCE IN OBSTETRICS AND GYNAECOLOGY SHIFTS HANDOVER - RE-AUDIT}

doi:10.1136/archdischild-2013-303966.259

A Yulia, C Pun, A Wijesiriwardana. North Cumbria NHS Trust, Carlisle, UK

\author{
Aims \\ 1. To assess the quality of effective handover practise and to \\ improve patients' care. \\ 2. To compare the handover practise with NHSLA guidelines. \\ 3. To access if audit recommendations have been addressed.
}

Background Handovers aim to convey high-quality and appropriate clinical information to oncoming healthcare professionals to allow for the safe transfer of responsibility for patients. Effective handover between shifts is vital to protect patient safety and assist doctors with clinical governance.

The NHSLA guideline aim to ensure effective communication within the multi-disciplinary team. This handover must include details of report tools for improving communication within the team.

Previous shifts handover audit performed in August 2011 showed that although $100 \%$ of medical staffs use the SBAR tools to aid the handover process, there was poor attendance of consultants and poor handover documentation. Recommendations were made to improve the quality of shifts handover.

Results A retrospective re-audit was performed during the month of February 2012. In the morning handover, $88 \%$ of medical staffs, $88 \%$ lead midwife and $63 \%$ consultants were present compared to $83 \%, 80 \%$ and $53 \%$ respectively in the previous audit. There is up to $27 \%$ increase of the attendance rates of health professional staffs in the evening handover. $93 \%$ of the handover process used the SBAR tools correctly compared to $87 \%$ previously. The documentations given all the midwives when transferring women from labour ward to postnatal ward met the requirements correctly. In conclusion, there is overall a good improvement in the attendances and documentation made during shifts handover.

\section{PL.78 MATERNAL REQUEST FOR A CAESAREAN SECTION IN THE ABSENCE OF MEDICAL INDICATIONS}

doi:10.1136/archdischild-2013-303966.260

A Sharpe, K Hinshaw. Newcastle University, Newcastle, UK

Introduction CS rates are rising worldwide. In the UK, $25 \%$ of women have a CS and 6-8\% of women express a preference. ${ }^{12}$

NICE recently updated guidelines stating that women can request a CS after they are fully informed of the risks. ${ }^{2}$ However the evidence of risks is of a poor quality, which questions how the opinions of doctors and midwives could influence women's decisions. $^{1}$
Methods A questionnaire-based study at the Sunderland Obstetric Department aimed to compare the views of pregnant women, midwives and doctors. Questionnaires used six different scenarios to ask firstly whether women should be able to request a CS and secondly the most common reason for the request. Further comparison was made between nulliparous and parous women. Data was analysed using excel.

\section{Results}

- There were 216 pregnant women, 56 midwives and 76 doctors.

- All groups favoured vaginal delivery for women who have had one or no previous deliveries.

- Twin pregnancy was considered a more acceptable indication.

- Nulliparous women were more willing for CS compared with parous women.

- All three groups agreed that fear of childbirth was the most common reason for women with no previous deliveries (patients 84 (38\%), midwives 32 (52\%) and doctors 34 (44\%).

- A higher proportion of patients and midwives, compared with doctors, considered concerns of their baby's safety (patients $78(39 \%)$, midwives $13(27 \%)$ and doctors $37(47 \%))$.

Discussion Womens' anxieties of childbirth need to be explored antenatally by healthcare professionals so that an informed decision can be made regarding the best mode of delivery for them.

\section{REFERENCES}

1. Mazzoni A, Althabe F, Liu N, et al. Women's preference for caesarean section: a sys temic review and meta analysis of observational studies. BGOG 2010;118:391-99.

2. NICE Clinical Guidelines. Caesarean Section, in Maternal Request for Caesarean Section 2011, Royal College of Obstetricians and Gynaecologists, p. 96-103.

\section{PL.79 A CLINICAL AUDIT; RISK OF SUBSEQUENT PERINEAL TRAUMA AFTER PREVIOUS OBSTETRIC ANAL SPHINCTER INJURY}

doi:10.1136/archdischild-2013-303966.261

E Bayar, F Mukri, K Ramalingam. Kingston Hospital, London, UK

Introduction Obstetric anal sphincter injuries (OASIS) are a serious complication of vaginal deliveries and can lead to faecal and urinary incontinence. Women, who have sustained OASIS in a previous pregnancy, undergo routine assessment to decide the appropriate mode of delivery in subsequent pregnancies. Many of these women are advised to, or opt for vaginal delivery.

Objective To evaluate the risk of subsequent perineal trauma in women who deliver vaginally following OASIS in previous delivery.

Methods This retrospective study analysed 88 women between April 2007 and April 2012 who had sustained anal sphincter damage during an index pregnancy and had a subsequent pregnancy and delivery.

Results 64 (72.8\%) had an instrumental delivery and 24 (27.2\%) had a spontaneous vaginal delivery (SVD) in their index pregnancy. In the instrumental delivery group, 21 (32.8\%) women had a subsequent Caesarean section delivery (18 elective Caesarean, 3 emergency Caesarean) while $43(67.2 \%)$ women had a vaginal delivery. In the SVD group, 8 (33.3\%) women had a Caesarean delivery (6 elective, 2 emergency) while 16 women (66.7\%) had a vaginal delivery. Out of the 59 women who had a vaginal delivery, 7 (11.9\%) sustained repeat $3^{\text {rd }}$ degree tear, $32(54.2 \%)$ had $2^{\text {nd }}$ degree tear, 7 (11.9\%) had episiotomy, 4 (6.8\%) had first degree tear, $9(15.2 \%)$ had intact perineum.

Discussion Most women with previous anal sphincter injury sustained a second degree tear while nearly $12 \%$ had a recurrent third degree tear. 